\title{
Notes on Certain Multivalent Analytic Functions Associated with a Linear Operator
}

\author{
Jin-Lin Liu \\ Department of Mathematics, Yangzhou University, Yangzhou, Jiangsu 225002, China \\ Correspondence should be addressed to Jin-Lin Liu; jlliu@yzu.edu.cn \\ Received 1 October 2012; Accepted 15 November 2012 \\ Academic Editor: Bao Qin Li
}

Copyright (C) 2013 Jin-Lin Liu. This is an open access article distributed under the Creative Commons Attribution License, which permits unrestricted use, distribution, and reproduction in any medium, provided the original work is properly cited.

The purpose of the present paper is to investigate some properties of multivalent analytic functions.

\section{Introduction}

Let $A_{p}(n)$ denote the class of the functions of the form

$$
f(z)=z^{p}+\sum_{k=n}^{\infty} a_{p+k} z^{p+k} \quad(n, p \in N=\{1,2,3, \ldots\})
$$

which are analytic in the open unit disk $U=\{z: z \in C$ and $|z|<1\}$. Also let the Hadamard product (or convolution) of two functions

$$
f_{j}(z)=z^{p}+\sum_{k=1}^{\infty} a_{k, j} z^{p+k} \quad(j=1,2)
$$

be given by

$$
\left(f_{1} * f_{2}\right)(z)=z^{p}+\sum_{k=1}^{\infty} a_{k, 1} a_{k, 2} z^{p+k}
$$

In [1], Liu introduced the following generalized Srivastava-Attiya operator $\mathbb{I}_{s, b}$ :

$$
\begin{gathered}
\mathbb{I}_{s, b}: A_{p}(1) \longrightarrow A_{p}(1) \\
\mathbb{I}_{s, b} f(z)=G_{p, s, b}(z) * f(z) \\
\left(z \in U ; b \in C \backslash Z_{0}^{-}, Z_{0}^{-}=\{0,-1,-2, \ldots\} ; s \in C\right),
\end{gathered}
$$

where

$$
\begin{gathered}
G_{p, s, b}(z)=(1+b)^{s}\left[\Phi_{p}(z, s, b)-b^{-s}\right], \\
\Phi_{p}(z, s, b)=\frac{1}{b^{s}}+\frac{z^{p}}{(1+b)^{s}}+\frac{z^{p+1}}{(2+b)^{s}}+\cdots .
\end{gathered}
$$

It is not difficult to see from (5) and (6) that

$$
\mathbb{I}_{s, b} f(z)=z^{p}+\sum_{k=1}^{\infty}\left(\frac{1+b}{k+1+b}\right)^{s} a_{p+k} z^{p+k}
$$

When $p=1$, the operator $\mathbb{I}_{s, b}$ is the well-known SrivastavaAttiya operator $\mathscr{L}_{s, b}$ [2]. The generalized Srivastava-Attiya operator has been studied by several authors (see [1-5]).

In this investigation, we focus on certain inequalities consisting of the following differential operator:

$$
\begin{aligned}
Q_{p, s, b}(\delta, \mu ; f)(z)= & \mu \frac{z\left(\mathbb{J}_{s, b} f(z)\right)^{\prime}}{\mathbb{J}_{s, b} f(z)} \\
& +\delta\left(1+\frac{z\left(\mathbb{J}_{s, b} f(z)\right)^{\prime \prime}}{\left(\mathfrak{J}_{s, b} f(z)\right)^{\prime}}\right) .
\end{aligned}
$$

Our results generalize the recent results obtained by Irmak et al. [6].

In order to prove our main results, we need the following lemmas. 
Lemma 1 (see [7]). Let $\Omega \subset C$ and suppose that the function $\psi: C^{2} \times U \rightarrow C$ satisfies $\psi\left(M e^{i \theta}, K e^{i \theta} ; z\right) \notin \Omega$ for all $K \geq$ $M n, \theta \in R$, and $z \in U$. If $h(z)=a+h_{n} z^{n}+\cdots$ is analytic in $U$ and $\psi\left(h(z), z h^{\prime}(z) ; z\right) \in \Omega$ for all $z \in U$, then $|h(z)|<M(z \in$ $U)$.

Lemma 2 (see [7]). Let $\Omega \subset C$ and suppose that the function $\psi: C^{2} \times U \rightarrow C$ satisfies $\psi(i x, y ; z) \notin \Omega$ for all $x \in R$, $y \leq-n\left(1+x^{2}\right) / 2$, and $z \in U$. If $h(z)=a+h_{n} z^{n}+\cdots$ is analytic in $U$ and $\psi\left(h(z), z h^{\prime}(z) ; z\right) \in \Omega$ for all $z \in U$, then $\operatorname{Re}\{h(z)\}>0(z \in U)$.

\section{Main Results}

We now state and then prove each of our main results given by Theorems 3 and 4 below.

Theorem 3. Let $f(z) \in A_{p}(n)$ with $\left(\mathbb{J}_{s, b} f(z)\right)^{\prime} \mathbb{J}_{s, b} f(z) /$ $z^{2 p-1} \neq 0$ for all $z \in U$, and also let $\delta \in R$ and $\mu \in R$. If

$$
\operatorname{Re}\left\{Q_{p, s, b}(\delta, \mu ; f)(z)\right\}<p(\mu+\delta)+\frac{n M}{M+1} \quad(z \in U),
$$

where $M \geq 1$, then

$$
\left|\left[\frac{\left(\mathbb{J}_{s, b} f(z)\right)^{\prime}}{p z^{p-1}}\right]^{\delta}\left[\frac{\mathbb{J}_{s, b} f(z)}{z^{p}}\right]^{\mu}-1\right|<M \quad(z \in U) .
$$

Proof. Put

$$
h(z)=\left[\frac{\left(\mathbb{J}_{s, b} f(z)\right)^{\prime}}{p z^{p-1}}\right]^{\delta}\left[\frac{\mathbb{J}_{s, b} f(z)}{z^{p}}\right]^{\mu}-1 .
$$

Then the function $h(z)$ is analytic in $U$ with $h(0)=0$. A simple computation shows that

$$
Q_{p, s, b}(\delta, \mu ; f)(z)=p(\mu+\delta)+\frac{z h^{\prime}(z)}{h(z)+1} .
$$

Now letting

$$
\begin{gathered}
\psi(r, s ; z)=p(\mu+\delta)+\frac{s}{r+1}, \\
\Omega=\left\{w \in C: \operatorname{Re}(w)<p(\mu+\delta)+\frac{n M}{M+1}\right\},
\end{gathered}
$$

we obtain that $\psi\left(h(z), z h^{\prime}(z) ; z\right)=Q_{p, s, b}(\delta, \mu ; f)(z) \in \Omega$ for all $z \in U$. Further, for any $\theta \in R, K \geq n M$, and $z \in U$, since $M \geq 1$, we also have

$$
\begin{aligned}
\operatorname{Re}\left\{\psi\left(M e^{i \theta}, K e^{i \theta} ; z\right)\right\} & =p(\mu+\delta)+K \operatorname{Re}\left(\frac{1}{M+e^{-i \theta}}\right) \\
& \geq p(\mu+\delta)+\frac{n M}{M+1},
\end{aligned}
$$

which shows that $\psi\left(M e^{i \theta}, K e^{i \theta} ; z\right) \notin \Omega$. Therefore, according to Lemma 1, we obtain $|h(z)|<M(z \in U)$. This completes the proof of Theorem 3.

Theorem 4. Let $f(z) \in A_{p}(n)$ with $\left(\mathbb{J}_{s, b} f(z)\right)^{\prime} \mathbb{J}_{s, b} f(z) /$ $z^{2 p-1} \neq 0$ for $z \in U$, and also let $\mu, \delta \in R$ and $\gamma \in[0,1)$. If

$$
\operatorname{Re}\left\{Q_{p, s, b}(\delta, \mu ; f)(z)\right\}>\beta(p, \delta, \mu ; \gamma) \quad(z \in U),
$$

where

$$
\beta(p, \delta, \mu ; \gamma)= \begin{cases}p(\mu+\delta)-\frac{n \gamma}{2(1-\gamma)}, & \gamma \in\left[0, \frac{1}{2}\right], \\ p(\mu+\delta)-\frac{n(1-\gamma)}{2 \gamma}, & \gamma \in\left[\frac{1}{2}, 1\right),\end{cases}
$$

then

$$
\operatorname{Re}\left\{\left[\frac{\left(\mathbb{J}_{s, b} f(z)\right)^{\prime}}{p z^{p-1}}\right]^{\delta}\left[\frac{\mathbb{J}_{s, b} f(z)}{z^{p}}\right]^{\mu}\right\}>\gamma \quad(z \in U) .
$$

Proof. Suppose that

$$
h(z)=\frac{1}{1-\gamma}\left(\left[\frac{\left(\mathbb{J}_{s, b} f(z)\right)^{\prime}}{p z^{p-1}}\right]^{\delta}\left[\frac{\mathbb{J}_{s, b} f(z)}{z^{p}}\right]^{\mu}-\gamma\right) .
$$

Then $h(z)=1+h_{n} z^{n}+\cdots$ is analytic in $U$. It is easily seen from (18) that

$$
Q_{p, s, b}(\delta, \mu ; f)(z)=p(\mu+\delta)+\frac{(1-\gamma) z h^{\prime}(z)}{(1-\gamma) h(z)+\gamma} .
$$

Further, since

$$
\begin{aligned}
& \psi(r, s ; z)=p(\mu+\delta)+\frac{(1-\gamma) s}{(1-\gamma) r+\gamma}, \\
& \Omega=\{w \in C: \operatorname{Re}(w)>\beta(p, \delta, \mu ; \gamma)\},
\end{aligned}
$$

it leads to $\psi\left(h(z), z h^{\prime}(z) ; z\right)=Q_{p, s, b}(\delta, \mu ; f)(z) \in \Omega$ for $z \epsilon$ $U$. Also, for any $x \in R, y \leq-n\left(1+x^{2}\right) / 2$ and $z \in U$, we have $\operatorname{Re}\{\psi(i x, y ; z)\}$

$$
\begin{aligned}
& =p(\mu+\delta)+\frac{\gamma(1-\gamma) y}{(1-\gamma)^{2} x^{2}+\gamma^{2}} \\
& \leq p(\mu+\delta)-\frac{n \gamma(1-\gamma)}{2} \frac{x^{2}+1}{(1-\gamma)^{2} x^{2}+\gamma^{2}} \equiv q(x) \\
& \leq \beta(p, \delta, \mu ; \gamma)= \begin{cases}\lim _{x \rightarrow+\infty} q(x), & \text { if } \gamma \in\left[0, \frac{1}{2}\right], \\
q(0), & \text { if } \gamma \in\left[\frac{1}{2}, 1\right),\end{cases}
\end{aligned}
$$

that is, $\psi(i x, y ; z) \notin \Omega$. Finally, by Lemma 2 , we obtain that $\operatorname{Re} h(z)>0(z \in U)$. The proof of Theorem 4 is completed.

Remark 5. For $p=1$ and $s=0$, Theorems 3 and 4 reduce to the results obtained by Irmak et al. [6]. 


\section{References}

[1] J.-L. Liu, "Subordinations for certain multivalent analytic functions associated with the generalized Srivastava-Attiya operator," Integral Transforms and Special Functions, vol. 19, pp. 893-901, 2008.

[2] H. M. Srivastava and A. A. Attiya, "An integral operator associated with the Hurwitz-Lerch Zeta function and differential subordination," Integral Transforms and Special Functions, vol. 18, pp. 207-216, 2007.

[3] N. E. Cho, I. H. Kim, and H. M. Srivastava, "Sandwich-type theorems for multivalent functions associated with the SrivastavaAttiya operator," Applied Mathematics and Computation, vol. 217, no. 2, pp. 918-928, 2010.

[4] G. Murugusundaramoorthy, "Subordination results for spirallike functions associated with the Srivastava-Attiya operator," Integral Transforms and Special Functions, vol. 23, no. 2, pp. 97-103, 2012.

[5] Z.-G. Wang, Z.-H. Liu, and Y. Sun, "Some properties of the generalized Srivastava-Attiya operator," Integral Transforms and Special Functions, vol. 23, no. 3, pp. 223-236, 2012.

[6] H. Irmak, T. Bulboacg, and N. Tuneski, "Some relations between certain classes consisting of $\alpha$-convex type and Bazilević type functions," Applied Mathematics Letters, vol. 24, pp. 2010-2014, 2011.

[7] S. S. Miller and P. T. Mocanu, Differential Subordinations, Theory and Applications, Marcel Dekker, New York, NY, USA, 2000. 


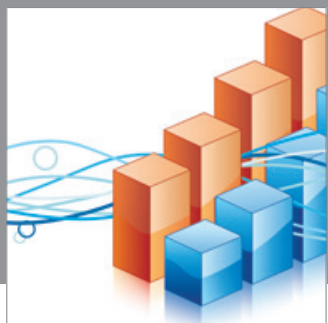

Advances in

Operations Research

mansans

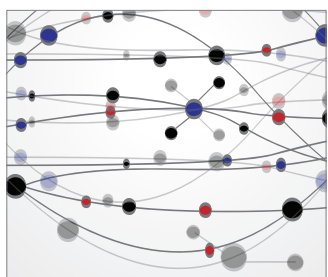

The Scientific World Journal
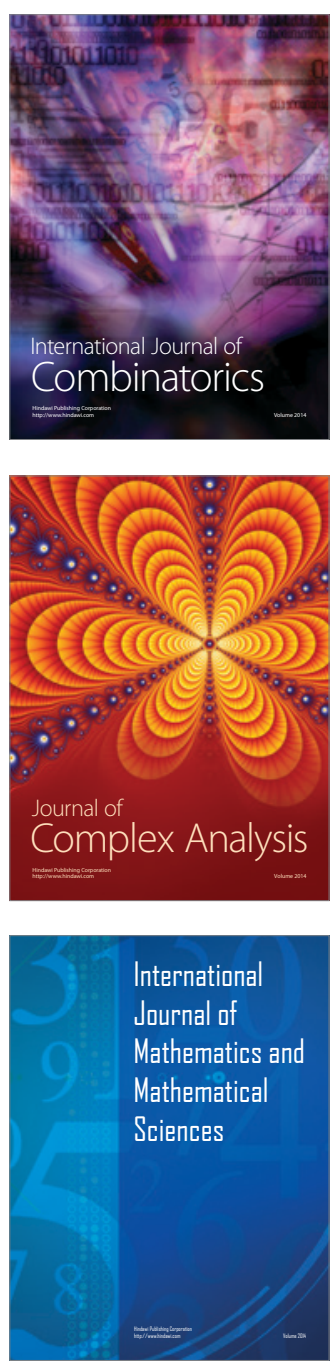
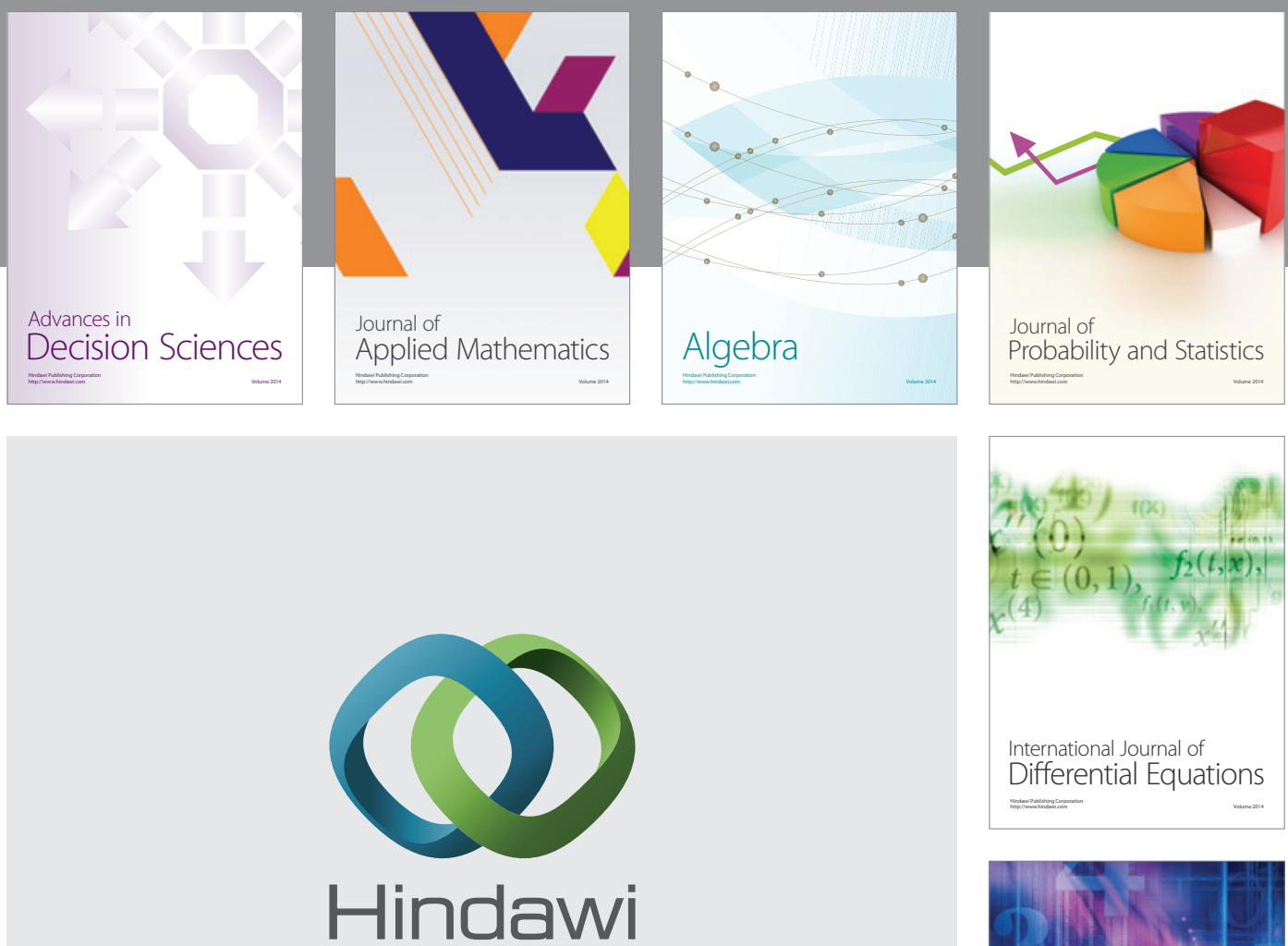

Submit your manuscripts at http://www.hindawi.com
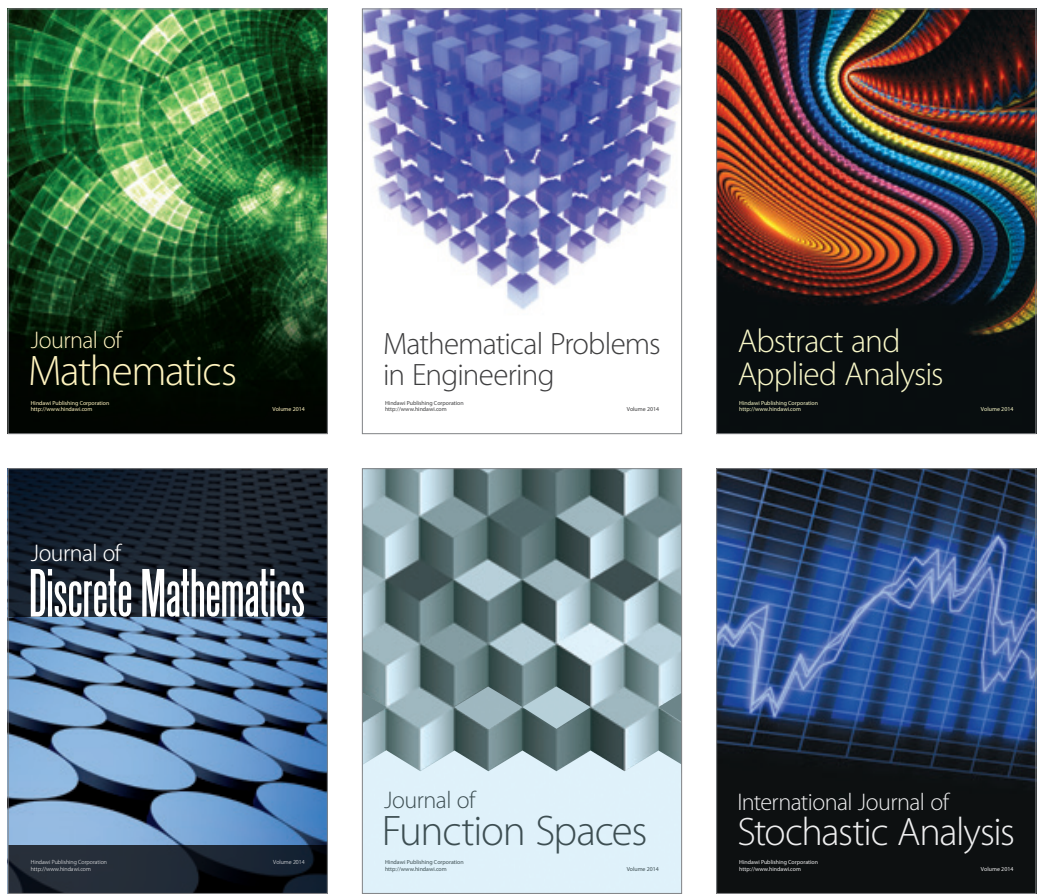

Journal of

Function Spaces

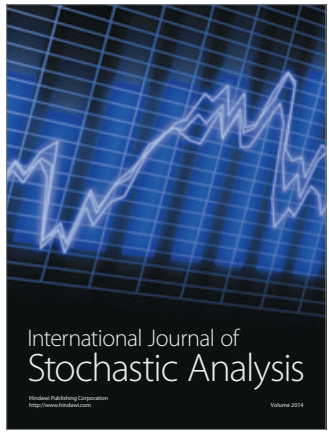

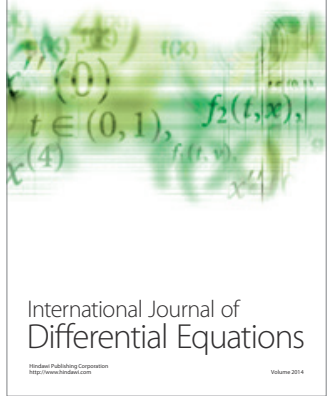
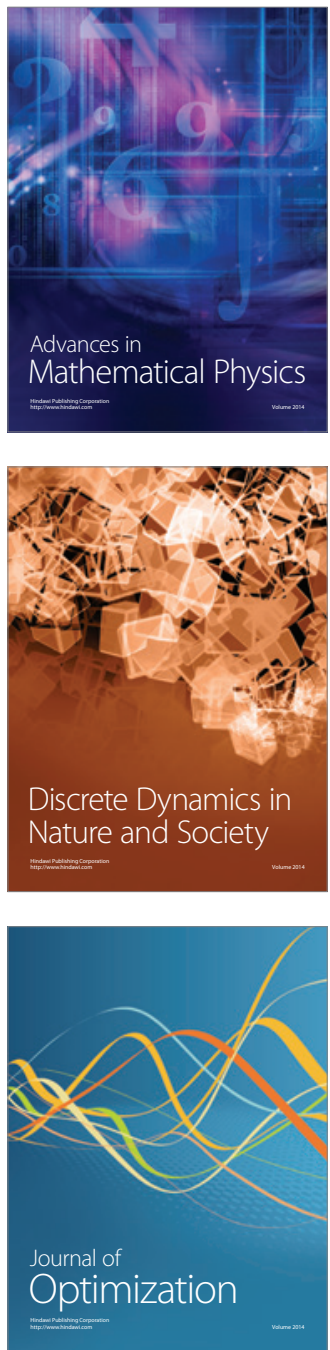